\title{
Faster extubation time with more stable hemodynamics during extubation and shorter total surgical suite time after propofol-based total intravenous anesthesia compared with desflurane anesthesia in lengthy lumbar spine surgery
}

\author{
Chueng-He Lu, MD, ${ }^{1}$ Zhi-Fu Wu, MD, ${ }^{1}$ Bo-Feng Lin, MD, ${ }^{1}$ Meei-Shyuan Lee, DrPH, ${ }^{2}$ Chin Lin, MPH, ${ }^{3}$ \\ Yuan-Shiou Huang, MD, ${ }^{1}$ and Yi-Hsuan Huang, MD' \\ 1Department of Anesthesiology, Tri-Service General Hospital and National Defense Medical Center; and ${ }^{2}$ School of Public Health
and ${ }^{3}$ Graduate Institute of Life Sciences, National Defense Medical Center, Taipei, Taiwan, Republic of China
}

OBJECTIVE Anesthesia techniques can contribute to the reduction of anesthesia-controlled time and may therefore improve operating room efficiency. However, little is known about the difference in anesthesia-controlled time between propofol-based total intravenous anesthesia (TIVA) and desflurane (DES) anesthesia techniques for prolonged lumbar spine surgery under general anesthesia.

METHODS A retrospective analysis was conducted using hospital databases to compare the anesthesia-controlled time of lengthy (surgical time > 180 minutes) lumbar spine surgery in patients receiving either TIVA via target-controlled infusion (TCI) with propofol/fentanyl or DES/fentanyl-based anesthesia, between January 2009 and December 2011. A variety of time intervals (surgical time, anesthesia time, extubation time, time in the operating room, postanesthesia care unit [PACU] length of stay, and total surgical suite time) comprising perioperative hemodynamic variables were compared between the 2 anesthesia techniques.

RESULTS Data from 581 patients were included in the analysis; 307 patients received TIVA and 274 received DES anesthesia. The extubation time was faster $(12.4 \pm 5.3$ vs $7.0 \pm 4.5$ minutes, $p<0.001)$, and the time in operating room and total surgical suite time was shorter in the TIVA group than in the DES group ( $326.5 \pm 57.2$ vs $338.4 \pm 69.4$ minutes, $p=$ 0.025 ; and $402.6 \pm 60.2$ vs $414.4 \pm 71.7$ minutes, $p=0.033$, respectively). However, there was no statistically significant difference in PACU length of stay between the groups. Heart rate and mean arterial blood pressure were more stable during extubation in the TIVA group than in the DES group.

CONCLUSIONS Utilization of TIVA reduced the mean time to extubation and total surgical suite time by 5.4 minutes and 11.8 minutes, respectively, and produced more stable hemodynamics during extubation compared with the use of DES anesthesia in lengthy lumbar spine surgery.

http://thejns.org/doi/abs/10.3171/2015.4.SPINE141143

KEY WORDS TIVA; propofol; desflurane; lengthy spine surgery; extubation; lumbar

$\mathrm{O}$ PERATING room efficiency is regulated by several factors; the 2 most important factors are anesthesiacontrolled time and turnover time. ${ }^{13}$ The interval between the end of surgery and extubation is of particular interest to surgeons and anesthesia care providers because it is affected by anesthetic agents. ${ }^{1,4,19,20}$ Anesthesiologists associate prolonged tracheal extubation time with poor re- covery from anesthesia. ${ }^{1}$ When surgeons score anesthesiologists' attributes on a scale from 0 ("not important") to 4 ("a factor that would make me switch groups/hospitals"), the average score for "patient quick to awaken" is 3.9. ${ }^{19}$

The majority of studies comparing the effects of different anesthesia regimens on operating room efficiency have tended to focus on relatively short and painless surgery

ABBREVIATIONS DES = desflurane; LOS = length of stay; MBP = mean blood pressure; $\mathrm{PACU}=$ postanesthesia care unit; $\mathrm{TCI}=$ target-controlled infusion; TIVA = total intravenous anesthesia.

SUBMITTED November 6, 2014. ACCEPTED April 21, 2015.

INCLUDE WHEN CITING Published online October 16, 2015; DOI: 10.3171/2015.4.SPINE141143. 
(e.g., day-case surgery). Recently, a meta-analysis comparing operating room recovery times for desflurane (DES) and propofol reported that, relative to propofol, DES proportionally reduced the mean time to extubation and time to follow commands ( $21 \%$ and $23 \%$, respectively). ${ }^{20}$ However, only 2 studies ${ }^{9,14}$ of prolonged anesthesia or surgery duration were included in this meta-analysis. In our previous prospective study, patients undergoing lengthy procedures were found to recover more quickly after total intravenous anesthesia (TIVA) via a target-controlled infusion (TCI) system, than with volatile anesthesia. ${ }^{2}$ The present study aimed to determine whether the use of TIVA with TCI is more effective than DES anesthesia in reducing anesthesia-controlled operating room time in patients undergoing lengthy lumbar spine surgery.

\section{Methods}

This study was approved by the ethics committee of Tri-Service General Hospital, Taipei, Taiwan. Medical records and electronic databases were collected from this hospital and were reviewed for all patients undergoing elective lengthy lumbar spine surgery (surgical time > 180 minutes), including posterolateral fusion and pedicle screw fixation (excluding patients with a deformity diagnosis), between January 2010 and December 2011. A total of 581 patients who received TIVA or DES anesthesia were included in this study. Exclusion criteria were patients younger than 18 years, emergency surgeries, combined propofol and DES anesthesia, non-DES inhalation anesthesia, failure to extubate, no postanesthesia care unit (PACU) stay, and incomplete data. Other parameters extracted from the electronic databases and medical records included demographic data, American Society of Anesthesiology physical status class, intraoperative fentanyl dosage, and perioperative hemodynamic parameters.

For the purposes of this study, the following times (in minutes) were calculated: 1) surgical time (incision to surgical completion and application of dressings), 2) anesthesia time (initiation of anesthesia to extubation), 3) extubation time (completion of surgery and dressing application to extubation), 4) time in the operating room (arrival in the operating room to departure from the operating room), 5) length of stay (LOS) in the PACU (time from arrival in the PACU to discharge from the PACU to the general ward), and 6) total surgical suite time (arrival in the operating room to discharge from the PACU to the general ward).

\section{Patient Groups}

There was no administration of medication prior to induction of anesthesia; however, regular monitoring, such as electrocardiography (lead II) and measurements of pulse oximetry, noninvasive blood pressure, respiratory rate, and end-tidal carbon dioxide pressure, were performed. The selection of anesthesia was based on the attending anesthesiologist's preference, and anesthesia management was performed by the same anesthesiologist according to clinical practice demands. In all patients, anesthesia was induced with propofol and fentanyl. The patients were then intubated and maintained with the anesthetics DES or propofol and the analgesic fentanyl.

\section{Anesthesia}

TIVA was induced with fentanyl $(2 \mu \mathrm{g} / \mathrm{kg})$ and lidocaine $(2 \%, 1.5 \mathrm{mg} / \mathrm{kg})$. Following this, continuous infusion of propofol (Fresenius 1\%) was initiated using a TCI system programmed with the Schneider model (Fresenius Orchestra Primea, Fresenius Kabi AG) at an effective target concentration of $4 \mu \mathrm{g} / \mathrm{ml}$. Rocuronium $\left(0.6 \mathrm{mg} \mathrm{kg}^{-1}\right)$ was administered when patients lost consciousness, followed by tracheal intubation. Anesthesia was maintained using TCI with propofol effective concentration 3-4 $\mu \mathrm{g} /$ $\mathrm{ml}$ and an oxygen flow of $0.3 \mathrm{~L} / \mathrm{min}$. Repetitive bolus injections of rocuronium and fentanyl were prescribed as required throughout the procedure.

In the DES group, anesthesia was induced with fentanyl $(2 \mu \mathrm{g} / \mathrm{kg})$, lidocaine $(2 \%, 1.5 \mathrm{mg} / \mathrm{kg})$, and propofol $(2 \mathrm{mg} /$ $\mathrm{kg})$. After loss of consciousness, rocuronium $(0.6 \mathrm{mg} / \mathrm{kg})$ was administered, and tracheal intubation was performed. Anesthesia was maintained with $8 \%-12 \%$ DES in an oxygen flow of $300 \mathrm{ml} / \mathrm{min}$ under a closed system without nitrous oxide, and repetitive bolus injections of rocuronium and fentanyl were prescribed as required throughout the procedure.

Maintenance of the effective concentration for the TCI propofol and DES concentrations was adjusted at a range of $0.2 \mu \mathrm{g} / \mathrm{ml}$ and $0.5 \%$, respectively, according to the hemodynamics. If 2 increments or decrements were unsuccessful in stabilizing the hemodynamics, the effective concentration ranges for the TCI propofol and DES concentrations were increased to $0.5 \mu \mathrm{g} / \mathrm{ml}$ and $2 \%$, respectively. The ventilation rate and maximum airway pressure were adjusted to maintain end-tidal carbon dioxide pressure at 35-45 mm Hg. Either cisatracurium (2 mg intravenously) or rocuronium (10 mg intravenously) was administered as required by the return of neuromuscular function.

At the end of the operation, DES or propofol was discontinued, and the lungs were ventilated with $100 \%$ oxygen at a fresh gas flow of $6 \mathrm{~L} / \mathrm{min}$. When the patient regained consciousness, with spontaneous and smooth respiration, the endotracheal tube was removed.

\section{Statistical Analysis}

Categorical and continuous variables were presented as proportions and means \pm SD, respectively. The differences in study variables between DES and TIVA were tested using the Student t-test or chi-square test where appropriate. In the multivariable analysis, patient characteristics (sex, age, height, and weight) were adjusted using linear regression analysis to obtain estimates of differences in all of the study variables. Since some study variables were the linear combination of each other, further analyses of individual variables were separated into 2 parts: 1) total surgical suite time, levels of fusion, and fentanyl dosage; and 2) anesthesia time, extubation time, PACU LOS, levels of fusion, and fentanyl dosage. These analyses were also adjusted according to the physiological state. The differences in heart rate and mean blood pressure (MBP) between the groups were also estimated. This study assumed that there were no interaction effects between any variables on any dependent variables. Statistical analyses were performed with R 3.0.1 software, and the level of statistical significance was determined as $\mathrm{p}<0.05$. 


\section{Results}

A total of 270 patients were excluded from the analysis, of whom 85 received combined inhalation anesthesia with propofol, 126 received sevoflurane anesthesia, and 59 had incomplete data (Fig. 1). Of the 581 patients included in this study, 307 received TIVA and 274 received DES anesthesia. There was no statistically significant difference in patient demographics between the groups, and the frequency of more than 2 levels of fusion was similar between them (Table 1).

Surgical and anesthesia time, and LOS in the PACU were not statistically significantly different between groups (Table 1). The extubation time was faster $(12.4 \pm$ 5.3 vs $7.0 \pm 4.5$ minutes, $\mathrm{p}<0.001)$, and the time in the operating room and surgical suites was shorter $(326.5 \pm$ 57.2 vs $338.4 \pm 69.4$ minutes, $p=0.025$; and $402.6 \pm 60.2$ vs. $414.4 \pm 71.7$ minutes, $\mathrm{p}=0.033$, respectively) in the TIVA group than in the DES group (Table 1). The dose of intraoperative fentanyl was significantly larger in the TIVA group than in the DES group $(268.2 \pm 61.4$ vs 228.5 $\pm 63.1 \mu \mathrm{g}, \mathrm{p}<0.001$; Table 1). Comparisons of surgical status by multiple variable analyses also demonstrated similar results between the groups (Table 2). Model 3 (Table 2) presents these results adjusted by other operating room times. Out of all the operating room times analyzed, the largest significant difference between the DES and TIVA groups was extubation time. Therefore, the differences in time in operating room and total surgical suite time between groups were mainly due to extubation time.

The heart rate and MBP during extubation in the TIVA group were lower than those in the DES group $(91.7 \pm 20.0$ vs $102.8 \pm 18.5 \mathrm{bpm}, \mathrm{p}<0.001$ and $108 \pm 17.3$ vs $115.9 \pm$ $13.9 \mathrm{~mm} \mathrm{Hg}, \mathrm{p}<0.001$, respectively; Table 1). Changes in heart rate and MBP in the TIVA group were also lower than those in the DES group $(17.1 \pm 20.1$ vs $29.5 \pm 18.5$ bpm, p $<0.001$ and $5.7 \pm 19.0$ vs $14.4 \pm 13.8 \mathrm{~mm} \mathrm{Hg}, \mathrm{p}$ $<0.001$, respectively; Table 1). Multivariable analyses revealed similar effects of physiological status between groups (Table 3).

To ensure unbiased results, the confounding effect of patient characteristics (sex, age, height, and weight), various time intervals, levels of fusion, and fentanyl dosage were investigated. The results revealed a similar frequency of more than 2 levels of fusion and no statistically significant difference in patient demographics between the DES and TIVA groups (Table 1). This indicated that the selection of anesthesia techniques was unaffected by levels of fusion. Furthermore, there were no intergroup interactive effects between any variables on any dependent variables for each time, and clinical characteristics were similar compared with the unadjusted results (Tables 2 and 3). These analyses adjusted for the physiological state and therefore guaranteed robust and unbiased results.

\section{Discussion}

In this retrospective study, propofol-based TIVA by TCI reduced the mean time to extubation (by 5.4 minutes) and total surgical suite time (by 11.8 minutes) and produced more stable hemodynamics during extubation, relative to DES anesthesia in patients undergoing lengthy lumbar spine surgery. These findings contradict the limited existing information obtained from previous randomized trials. However, they importantly reveal that extubation time and total surgical suite time significantly differ according to duration of surgery and anesthetic drug selection.

Reductions in operating room time reduce direct labor costs either when the operating room has overused time or when there is more than 8 hours of appropriate staffing planned for the operating room and the staffing can be reduced to 8 hours. ${ }^{6,17,18}$ Facilities that consistently use operating rooms for more than 8 hours daily will benefit from the largest direct cost reductions. Among these operating rooms, each 1-minute reduction in operating room time

\section{Lengthy lumbar surgery $(n=851)$}

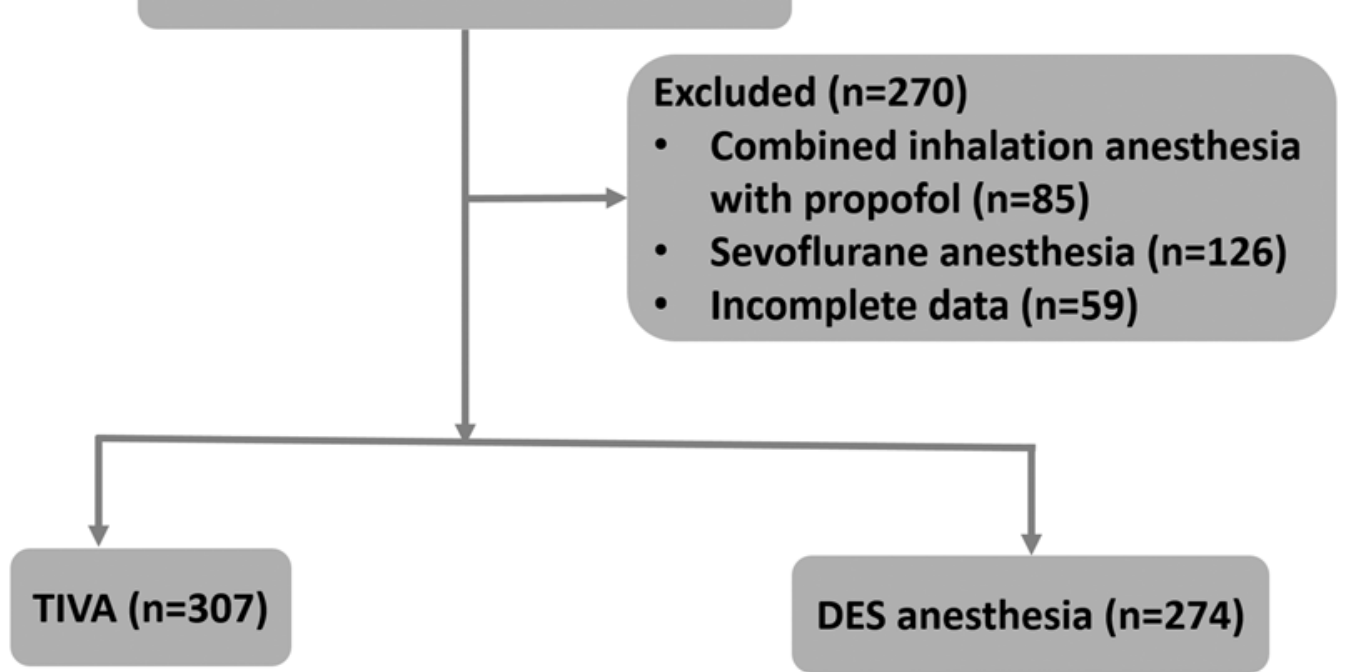

FIG. 1. Study protocol. 
TABLE 1. Patient characteristics and various time intervals in both groups*

\begin{tabular}{|c|c|c|c|}
\hline Variable & DES $(n=274)$ & $\operatorname{TIVA}(n=307)$ & $\mathrm{p}$ Value \\
\hline Female sex & $45.3 \%$ & $46.3 \%$ & 0.875 \\
\hline Mean age, yrs & $59.9 \pm 10.0$ & $63.8 \pm 15.4$ & 0.757 \\
\hline Mean height, $\mathrm{cm}$ & $164.5 \pm 9.0$ & $164.4 \pm 8.9$ & 0.919 \\
\hline Mean weight, kg & $70.2 \pm 12.6$ & $70.3 \pm 12.5$ & 0.903 \\
\hline Mean arrival in OR to start of anesthesia, mins & $9.0 \pm 3.9$ & $7.9 \pm 3.9$ & 0.001 \\
\hline Mean surgical time, mins & $253.1 \pm 64.1$ & $254.5 \pm 53.0$ & 0.775 \\
\hline Mean anesthesia time, mins & $306.6 \pm 68.4$ & $302.3 \pm 56.3$ & 0.417 \\
\hline Mean extubation time, mins & $12.4 \pm 5.3$ & $7.0 \pm 4.5$ & $<0.001$ \\
\hline Mean extubation to departure from OR, min & $10.5 \pm 5.4$ & $9.3 \pm 4.9$ & 0.004 \\
\hline Mean time in OR, mins & $338.4 \pm 69.4$ & $326.5 \pm 57.2$ & 0.025 \\
\hline Mean PACU LOS, mins & $76.0 \pm 15.8$ & $76.0 \pm 16.8$ & 0.953 \\
\hline Mean total surgical suite time, mins & $414.4 \pm 71.7$ & $402.6 \pm 60.2$ & 0.033 \\
\hline Mean fentanyl, $\mu \mathrm{g}$ & $228.5 \pm 63.1$ & $268.2 \pm 61.4$ & $<0.001$ \\
\hline Mean baseline HR, bpm & $73.3 \pm 14.3$ & $74.6 \pm 15.3$ & 0.299 \\
\hline Mean baseline MBP, $\mathrm{mm} \mathrm{Hg}$ & $101.4 \pm 11.9$ & $102.4 \pm 10.8$ & 0.337 \\
\hline Mean max HR during extubation, bpm & $102.8 \pm 18.5$ & $91.7 \pm 20.0$ & $<0.001$ \\
\hline Mean max MBP during extubation, $\mathrm{mm} \mathrm{Hg}$ & $115.9 \pm 13.9$ & $108 \pm 17.3$ & $<0.001$ \\
\hline Mean $\Delta \mathrm{HR}, \mathrm{bpm}$ & $29.5 \pm 18.5$ & $17.1 \pm 20.1$ & $<0.001$ \\
\hline Mean $\triangle \mathrm{MBP}, \mathrm{mm} \mathrm{Hg}$ & $14.4 \pm 13.8$ & $5.7 \pm 19.0$ & $<0.001$ \\
\hline PLF \& PSF, >2 levels & $32.5 \%$ & $34.9 \%$ & 0.546 \\
\hline
\end{tabular}

$\mathrm{HR}=$ heart rate; $\mathrm{MBP}=$ mean blood pressure; $\triangle \mathrm{HR}=$ maximal heart rate during extubation - baseline heart rate; $\triangle \mathrm{MBP}=$ maximal $\mathrm{MBP}$ during extubation - baseline MBP; OR = operating room; PLF = posterolateral fusion; PSF = pedicle screw fixation.

* The baseline patient data indicate the measurements before the start of anesthesia. Mean data are shown as mean \pm SD.

$\dagger p$ values $<0.05$ were considered significant.

results in an overall 1.1- to 1.2-minute reduction in regularly scheduled labor costs.${ }^{6,7}$ Consequently, small reductions in operating room time, achieved through the reduction of extubation and total surgical suite time, as reported in this study (in which the operating room workday in this study exceeded 8 hours), can be reasonably considered to be of economic benefit. Additionally, an intangible value of time saved may be achieved from more predictable recovery (e.g., fewer frustrated surgeons complaining of delays in beginning the next case).

Differences in operating room recovery times between anesthetic agents have been extensively studied because they can limit operating room throughput; this is based on data showing that nonanesthesia operating room personnel must wait for the patient to be extubated during emergence from anesthesia in the majority of cases $(>66 \%){ }^{8,16}$ Previously, Masursky et al. ${ }^{17}$ demonstrated that longer times to extubation are associated with an increased risk of at least one person waiting or being idle in the operating room (slowing the workflow). In addition, cases with prolonged tracheal extubation times have longer times from operating room exit to the start of the surgeon's next case in the operating room. ${ }^{4}$ Therefore, selection of an anesthetic technique associated with faster extubation is associated with rapid operating room workflow and reductions in the time from operating room exit to the start of the surgeon's next case. ${ }^{4,17}$ An anesthesia technique with shorter extubation times would decrease waiting time for the operating room staff and reduce the time from the end of surgery to operating room exit. ${ }^{5}$

Extubation times differ significantly among anesthetic drugs. ${ }^{4,15,20}$ Propofol has become popular for general anesthesia, especially in the ambulatory setting. It is frequently used in combination with remifentanil since both drugs have been reported to enable rapid emergence from anesthesia and early return to normal activities. ${ }^{10,15}$ Until recently, remifentanil was unavailable in Taiwan. However, the duration of emergence from anesthesia has been shown to be significantly shorter with TIVA than with inhalation anesthesia in breast cancer and gynecological laparoscopic surgeries. ${ }^{3,12}$ In addition, in a previous prospective study, a propofol and fentanyl combination was found to be cost-saving and resulted in faster emergence and extubation in lengthy spinal surgery when compared with DES and sevoflurane anesthesia. ${ }^{2}$ Furthermore, the use of TIVA has been shown to reduce the mean time to extubation by at least $9 \%$ and duration in PACU by more than $1 \%$ compared with the use of DES anesthesia in ophthalmic surgery. ${ }^{22}$

In this retrospective study of patients undergoing lengthy lumbar spine surgery, propofol-based TIVA by TCI was also found to reduce the mean time to extubation and total surgical suite time relative to DES. Our findings contradict those of a recent meta-analysis ${ }^{20}$ comparing the operating room recovery time of DES with that of propofol. The discrepancy in these findings is most likely due to differences 

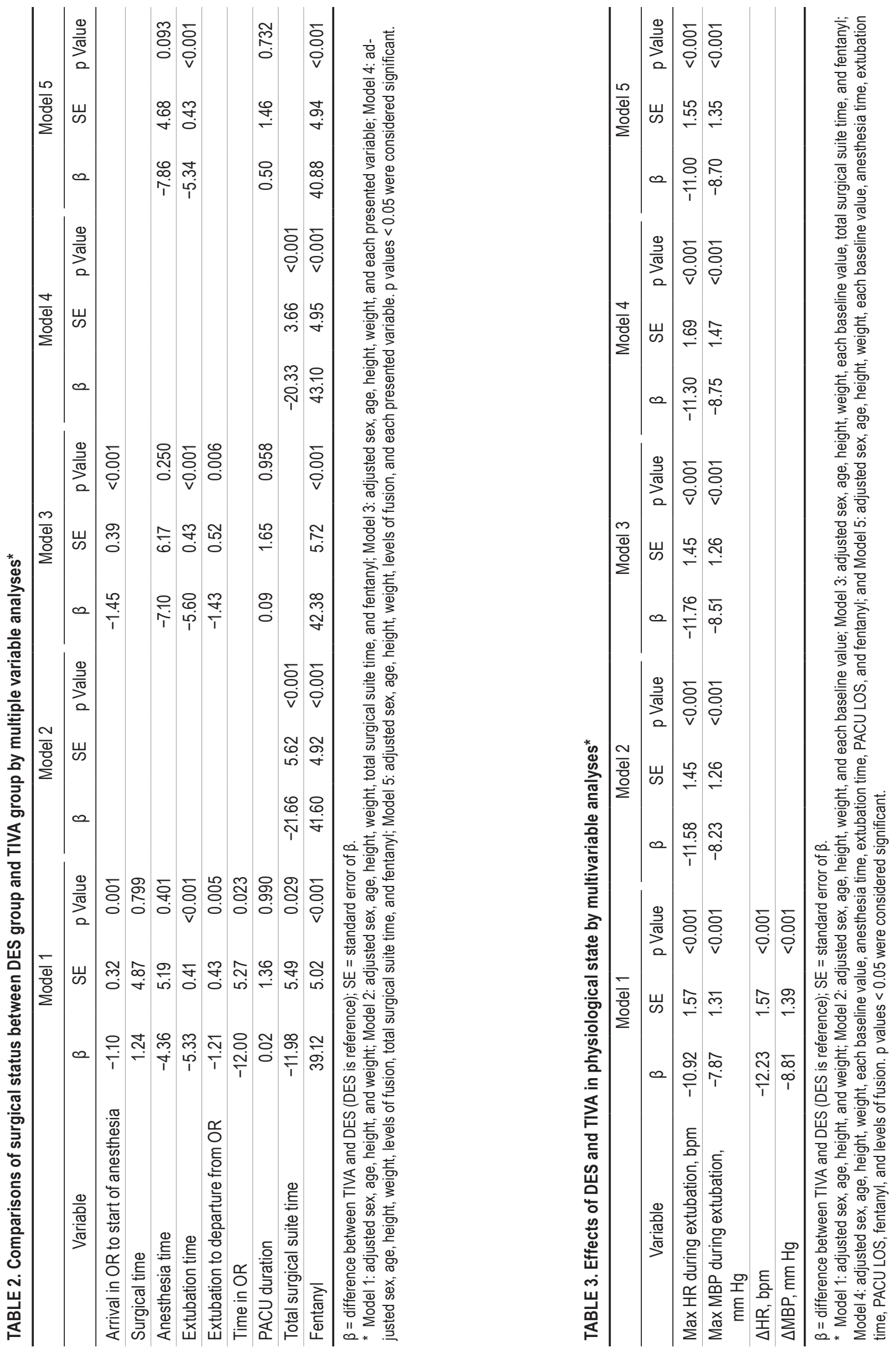
in anesthesia drugs and surgical procedure (short-duration surgical procedures were predominately investigated in the previous meta-analysis).

The hemodynamics investigated in this study were more stable during extubation. During extubation, heart rate and MBP increased less in the TIVA group than in the DES group. This may be explained because of the use of propofol and the larger dose of fentanyl administered during operations, which reduced stimulation during extubation. Hohlrieder et al. ${ }^{11}$ found that TIVA is associated with significantly less coughing and reduces hemodynamic response when compared with inhalation anesthesia in elective lumbar disc surgery during emergence from anesthesia. In addition, Wang et al. ${ }^{21}$ demonstrated more stable hemodynamics in the TCI patient group and significantly lower mean arterial pressure and faster recovery profiles compared with the fentanyl/DES patient group. Variable doses of intraoperative fentanyl administrated between groups may explain these differences in findings.

A retrospective study design may lead to bias regarding standardization and comparability of study groups. For the purpose of this study, retrospective analysis of data provided a major advantage since anesthesia management was performed by the attending anesthesiologist, according to clinical demands, as opposed to being determined by a study protocol. This study, performed under real clinical conditions, more accurately reflects the clinically relevant benefits that may be expected with the use of new drugs or devices. Finally, comorbidity may play a role in extubation time; however, this could not be investigated in this study owing to lack of patient comorbidity records in the hospital databases.

\section{Conclusions}

Relative to DES, propofol-based TIVA by TCI reduced the mean time to extubation and total surgical time by 5.4 and 11.8 minutes, respectively, and produced more stable hemodynamics during extubation in lengthy lumbar spine surgery. The reduction in extubation and total surgical suite time will have an economic impact through the associated increases in operating room productivity and reduction in labor costs, especially when operating rooms are consistently used for more than 8 hours daily.

\section{References}

1. Apfelbaum JL, Grasela TH, Hug CC Jr, McLeskey CH, Nahrwold ML, Roizen MF, et al: The initial clinical experience of 1819 physicians in maintaining anesthesia with propofol: characteristics associated with prolonged time to awakening. Anesth Analg 77 (4 Suppl):S10-S14, 1993

2. Chan SM, Horng HC, Huang ST, Ma HI, Wong CS, Cherng $\mathrm{CH}$, et al: Drug cost analysis of three anesthetic regimens in prolonged lumbar spinal surgery. J Med Sci 29:75-80, 2009

3. Chen JL, Kuo CP, Chen YF, Chen YW, Yu JC, Lu CH, et al: Do anesthetic techniques affect operating room efficiency? Comparison of target-controlled infusion of propofol and desflurane anesthesia in breast cancer surgery. J Med Sci 33:205-210, 2013

4. Dexter F, Bayman EO, Epstein RH: Statistical modeling of average and variability of time to extubation for metaanalysis comparing desflurane to sevoflurane. Anesth Analg 110:570-580, 2010
5. Dexter F, Epstein RH: Increased mean time from end of surgery to operating room exit in a historical cohort of cases with prolonged time to extubation. Anesth Analg 117:14531459,2013

6. Dexter F, Epstein RH: Typical savings from each minute reduction in tardy first case of the day starts. Anesth Analg 108:1262-1267, 2009

7. Dexter F, Macario A, Manberg PJ, Lubarsky DA: Computer simulation to determine how rapid anesthetic recovery protocols to decrease the time for emergence or increase the phase I postanesthesia care unit bypass rate affect staffing of an ambulatory surgery center. Anesth Analg 88:1053-1063, 1999

8. Dexter F, Marcon E, Aker J, Epstein RH: Numbers of simultaneous turnovers calculated from anesthesia or operating room information management system data. Anesth Analg 109:900-905, 2009

9. Grottke O, Dietrich PJ, Wiegels S, Wappler F: Intraoperative wake-up test and postoperative emergence in patients undergoing spinal surgery: a comparison of intravenous and inhaled anesthetic techniques using short-acting anesthetics. Anesth Analg 99:1521-1527, 2004

10. Grundmann U, Silomon M, Bach F, Becker S, Bauer M, Larsen B, et al: Recovery profile and side effects of remifentanilbased anaesthesia with desflurane or propofol for laparoscopic cholecystectomy. Acta Anaesthesiol Scand 45:320-326, 2001

11. Hohlrieder M, Tiefenthaler W, Klaus H, Gabl M, Kavakebi P, Keller C, et al: Effect of total intravenous anaesthesia and balanced anaesthesia on the frequency of coughing during emergence from the anaesthesia. Br J Anaesth 99:587-591, 2007

12. Horng HC, Kuo CP, Ho CC, Wong CS, Yu MH, Cherng CH, et al: Cost analysis of three anesthetic regimens under auditory evoked potentials monitoring in gynecologic laparoscopic surgery. Acta Anaesthesiol Taiwan 45:205-210, 2007

13. Junger A, Klasen J, Hartmann B, Benson M, Röhrig R, Kuhn $\mathrm{D}$, et al: Shorter discharge time after regional or intravenous anaesthesia in combination with laryngeal mask airway compared with balanced anaesthesia with endotracheal intubation. Eur J Anaesthesiol 19:119-124, 2002

14. Juvin P, Servin F, Giraud O, Desmonts JM: Emergence of elderly patients from prolonged desflurane, isoflurane, or propofol anesthesia. Anesth Analg 85:647-651, 1997

15. Lebenbom-Mansour MH, Pandit SK, Kothary SP, Randel GI, Levy L: Desflurane versus propofol anesthesia: a comparative analysis in outpatients. Anesth Analg 76:936-941, 1993

16. Marcon E, Dexter F: An observational study of surgeons' sequencing of cases and its impact on postanesthesia care unit and holding area staffing requirements at hospitals. Anesth Analg 105:119-126, 2007

17. Masursky D, Dexter F, Kwakye MO, Smallman B: Measure to quantify the influence of time from end of surgery to tracheal extubation on operating room workflow. Anesth Analg 115:402-406, 2012

18. McIntosh C, Dexter F, Epstein RH: The impact of servicespecific staffing, case scheduling, turnovers, and first-case starts on anesthesia group and operating room productivity: a tutorial using data from an Australian hospital. Anesth Analg 103:1499-1516, 2006

19. Vitez TS, Macario A: Setting performance standards for an anesthesia department. J Clin Anesth 10:166-175, 1998

20. Wachtel RE, Dexter F, Epstein RH, Ledolter J: Meta-analysis of desflurane and propofol average times and variability in times to extubation and following commands. Can J Anaesth 58:714-724, 2011

21. Wang Y, Yan M, He JG, Zhu YM, Hu XS, Li X, et al: A randomized comparison of target-controlled infusion of remifentanil and propofol with desflurane and fentanyl for 
laryngeal surgery. ORL J Otorhinolaryngol Relat Spec 73 (1 Spec):47-52, 2011

22. Wu ZF, Jian GS, Lee MS, Lin C, Chen YF, Chen YW, et al: An analysis of anesthesia-controlled operating room time after propofol-based total intravenous anesthesia compared with desflurane anesthesia in ophthalmic surgery: a retrospective study. Anesth Analg 119:1393-1406, 2014

\section{Disclosure}

The authors report no conflict of interest concerning the materials or methods used in this study or the findings specified in this paper.

\section{Author Contributions}

Conception and design: YH Huang, Lu, Wu. Acquisition of data: YH Huang. Analysis and interpretation of data: Lee, C Lin. Drafting the article: YH Huang, Lu. Critically revising the article: $\mathrm{Lu}, \mathrm{Wu}, \mathrm{BF}$ Lin. Reviewed submitted version of manuscript: YH Huang, Lu, Wu, BF Lin, YS Huang. Approved the final version of the manuscript on behalf of all authors: YH Huang. Statistical analysis: Lee, C Lin. Study supervision: Wu.

\section{Correspondence}

Yi-Hsuan Huang, \#325, Section 2, Chenggung Rd., Neihu 114, Taipei, Taiwan, Republic of China. email: yixiun@hotmail.com. 